\title{
Epigenetic Inactivation of TRAIL Decoy Receptors at 8p I 2-2 I.3 Commonly Deleted Region Confers Sensitivity to Apo2L/TRAIL-Cisplatin Combination Therapy in Cervical Cancer
}

\author{
Gopeshwar Narayan,,,$\dagger$ Dongxu Xie, ${ }^{2}$ Ganchimeg Ishdorj, ${ }^{2}$ Luigi Scotto, ${ }^{1,3}$ Mahesh Mansukhani, \\ Bhavana Pothuri, ${ }^{4, \$}$ Jason D. Wright, ${ }^{4}$ Andreas M. Kaufmann, ${ }^{5}$ Achim Schneider, ${ }^{5}$ \\ Hugo Arias-Pulido, ${ }^{6}$ and Vundavalli V. Murty ${ }^{1,2 *}$ \\ 'Department of Pathology and Cell Biology, Columbia University Medical Center, New York, NY \\ ${ }^{2}$ Institute for Cancer Genetics, Herbert Irving Comprehensive Cancer Center, Columbia University Medical Center, New York, NY \\ ${ }^{3}$ Department of Medicine, Columbia University Medical Center, New York, NY \\ ${ }^{4}$ Gynecologic Oncology, Columbia University Medical Center, New York, NY \\ ${ }^{5}$ Department of Gynecology, Charité-Universitätsmedizin Berlin, Berlin, Germany \\ ${ }^{6}$ Department of Radiology, Geisel School of Medicine at Dartmouth College, Lebanon, $\mathrm{NH}$
}

Multiple chromosomal regions are affected by deletions in cervical cancer (CC) genomes, but their consequence and target gene involvement remains unknown. Our single nucleotide polymorphism (SNP) array identified 8p copy number losses localized to an $8.4 \mathrm{Mb}$ minimal deleted region (MDR) in 36\% of CC. The 8p MDR was associated with tumor size, treatment outcome, and with multiple HPV infections. Genetic, epigenetic, and expression analyses of candidate genes at MDR identified promoter hypermethylation and/or inactivation of decoy receptors TNFRSFIOC and TNFRSFIOD in the majority of CC patients. TNFRSFIOC methylation was also detected in precancerous lesions suggesting that this change is an early event in cervical tumorigenesis. We further demonstrate here that CC cell lines exhibiting downregulated expression of TNFRSFIOC and/or TNFRSFIOD effectively respond to TRAIL-induced apoptosis and this affect was synergistic in combination with DNA damaging chemotherapeutic drugs. We show that the CC cell lines harboring epigenetic inactivation of TRAIL decoy receptors effectively activate downstream caspases suggesting a critical role of inactivation of these genes in efficient execution of extrinsic apoptotic pathway and therapy response. Therefore, these findings shed new light on the role of genetic/epigenetic defects in TRAIL decoy receptor genes in the pathogenesis of CC and provide an opportunity to explore strategies to test decoy receptor gene inactivation as a biomarker of response to Apo2L/TRAIL-combination therapy. (c) 2015 Wiley Periodicals, Inc.

\section{INTRODUCTION}

Genetic studies identified the short arm of chromosome $8(8 p)$ as a frequent target of nonrandom and recurrent deletion in cervical cancer (CG) (Mitra et al., 1994; Hampton et al., 1996; Harris et al., 2003; Bhattacharya et al., 2004; Rao et al., 2004; Kloth et al., 2007; Ojesina et al., 2014). These studies suggest the presence of tumor suppressor genes on $8 \mathrm{p}$ and their involvement in the development of CC. However, until now the biological impact or the relevant deregulated genes and the mechanisms underlying $8 \mathrm{p}$ deletion has not been known. 8 p deletions also have been frequently implicated in multiple other tumor types including prostate, liver, kidney, bladder, and colorectal carcinomas (Chang et al., 2007; Beroukhim et al., 2009; Midorikawa et al., 2009; Williams et al., 2010), and a number of tumor suppressor genes have been suggested such as NKX3-1, tumor necrosis factor-related family of genes (TNFRSF10C, TNFRSF10D), NRG1, UNC5D, ARHGEF10, DLC1 (Macartney-Coxson et al., 2008; Xue et al., 2008; Chua et al., 2009),

Additional Supporting Information may be found in the online version of this article.

Grant sponsor: National Institutes of Health; Grant number: CA095647.

*Correspondence to: Vundavalli V. Murty; Irving Cancer Research Center, Columbia University Medical Center, 1130 St. Nicholas Avenue, New York, New York 10032. E-mail: vvm2@ columbia.edu

†Present address: Department of Molecular \& Human Genetics, Banaras Hindu University, India

${ }^{\ddagger}$ Present address: New York University Cancer Institute, NYU Langone Medical Center, New York, NY

Received 31 August 2015; Revised 30 September 2015;

Accepted 6 October 2015

DOI 10.1002/gcc.22325

Published online 6 November 2015 in

Wiley Online Library (wileyonlinelibrary.com). 
indicating that one or more genes residing on $8 p$ are commonly involved in a wide-variety of tumor types.

Despite the advances in early detection, CG remains a major cause of cancer deaths in women worldwide due to treatment failure of invasive cancer (Waggoner, 2003). CG progresses by distinct morphological changes from normal epithelium to carcinoma through grades of squamous intraepithelial lesions (SILs). While infection of high-risk human papilloma viruses (HPVs) is recognized as an important etiologic agent in cervical pathogenesis, other genetic alterations are needed for the progression (zur Hausen, 2002). To date, no genetic markers are available to predict response to treatment of invasive CC. Therefore, identification of critical "somatic genetic hits" in $\mathrm{CC}$ is important in understanding its biology and establishing clinically relevant biomarkers.

In this study, we identified recurrent $8 \mathrm{p}$ deletions in invasive $\mathrm{CC}$ and inactivation of decoy receptors TNFRSF10C and TNFRSF1OD as major target genes at this region. We demonstrate that inactivation of decoy receptors elicits synergistic TRAIL-Cisplatin induced cell death through extrinsic apoptotic pathway in the presence of DNA-damaging drugs. Additionally, the promoter hypermethylation of TNFRSF1OC occurs very early during the tumor progression suggesting a role for this gene in CG development.

\section{MATERIALS AND METHODS}

\section{Patients, Tumor Tissues, and Cell Lines}

A total of 264 samples of DNA representing various stages of CC progression were used in this study. These include 123 invasive CG (nine cell lines and 114 primary tumors), 141 cytological pap smears from normal and precancerous lesions. The cell lines HT-3, ME-180, CaSki, MS751, C-4I, C33A, SW756, HeLa, and SiHa were obtained from American Type Culture Collection (ATCG) and grown in culture as per the supplier's specifications. All specimens were obtained from Columbia University Medical Center (NY), Instituto Nacional de Cancerología (Bogota, Colombia), and the Department of Gynecology of Campus Benjamin Franklin, Charité-Universitätsmedizin Berlin (Germany) with appropriate informed consent and approval of protocols by institutional review boards (Narayan et al., 2003b). All primary tumors were diagnosed as squamous cell carcinoma but six that were diagnosed as adenocarcinoma. Clini- cal information such as age, stage, and size of the tumor, follow-up data after initial diagnosis and treatment were obtained for the majority of tumors from the review of institutional medical records. Tissues were frozen at $-80^{\circ} \mathrm{C}$ immediately after resection and were embedded with tissue freeze medium (O'TG) before macrodissection. All primary tumor specimens were determined to contain at least $60 \%$ tumor by examination of hematoxylin and eosin staining of adjacent sections. Cytological specimens were collected and processed as reported previously (Narayan et al., 2009). Thirty-four pap smears diagnosed as normal, 22 as atypical squamous cells of undetermined significance (ASC-US), 43 as low-grade SIL (LSIL), and 42 as high-grade SIL (HSIL) were analyzed. The diagnosis of all HSILs was also confirmed by a biopsy. High-molecular weight DNA from frozen tumor tissues, cell lines, and cell pellets from cytology smears was isolated by standard methods. HPV types were identified as described earlier (Narayan et al., 2003a).

\section{SNP Array and Fluorescence In Situ Hybridization Analysis}

The Affymetrix 250K NspI SNP chip was used for copy number analysis as per the manufacturer's protocol. SNP array was performed on $80 \mathrm{CG}$ specimens ( 9 cell lines and 71 primary tumors) selected based on high tumor content and 7 macrodissected normal cervical squamous epithelia as controls. Acquisition and analysis of copy number data for chromosome 8 using CytoBand information files from the dChip website (http://biosun1. harvard.edu/complab/dchip/chromosome.htm\#refgene) was performed as described (Scotto et al., 2008a,b). Copy numbers $<1.5$ were considered as deletion, 2.5-4.0 as gain, and $>4.1$ as amplification in the raw copy number view.

Affymetrix U133A array (Santa Clara, CA) was hybridized using RNA from 42 CG cases (33 primary tumors enriched by macrodissection and 9 cell lines) and 20 macrodisssected normal cervical squamous epithelial cells using the standard protocols (Li and Wong, 2001; Lin et al., 2004; Scotto et al., 2008a; Scotto et al., 2008b) (GEO accession numbers: GSE9750 and GSE10092). A total of 671 probe sets on Chr8 were present in U133A array representing $6 \%$ of the genome $(2.4 \%$ on $8 \mathrm{p})$. To obtain differentially expressed gene signatures, we compared all normal with all tumor samples using the criteria of 1.75 -fold change between the group means at $90 \%$ confidence interval and a 
TABLE I. Frequency of Promoter Hypermethylation of Decoy Receptor Genes in CC Progression

\begin{tabular}{lcccc}
\hline & \multicolumn{2}{c}{ TNFRSFIOC } & \multicolumn{2}{c}{ TNFRSFIOD } \\
\cline { 2 - 5 } Tissue type & No. studied & Methylated (\%) & No. studied & Methylated (\%) \\
\hline Normal cervix & 34 & 0 & ND & ND \\
ASC-US & 22 & $5(22.7)$ & ND & ND \\
LSIL & 43 & $8(I 8.6)$ & ND & ND \\
HSIL & 42 & $5(11.9)$ & 114 & ND \\
Primary invasive cancer & 114 & $48(42.1)$ & 9 & $5(4.4)$ \\
CC cell lines & 9 & $8(88.9)$ & 123 & $2(22.2)$ \\
All Invasive cancer & 123 & $56(45.5)$ & $7(5.7)$ \\
\hline
\end{tabular}

ASC-US, atypical squamous cells of undetermined significance; LSIL, low-grade squamous intraepithelial lesion; HSIL, high-grade squamous intraepithelial lesion; ND, not done.

significance level of $P<0.05$. All negative expression values for each probe set were truncated to 1 before calculating fold changes and $<10 \%$ of samples with present call in each group were excluded. The differentially expressed probe list was used in all subsequent supervised analyses using the same criteria between various groups to obtain relevant gene signatures.

Fluorescence in situ hybridization (FISH) analysis was performed by standard methods using BAC clones (RP11-875O11 and RP11-109B10) that span the $8 \mathrm{p} 21$ common region of deletion and centromere 8 as control.

\section{Methylation-Specific polymerase chain reaction (PCR) and Sequencing}

Tumor and control DNAs were treated with sodium bisulphite as described (Narayan et al., 2003a). Primer sets used for amplification of methylated and unmethylated DNA spanning the $\mathrm{CpG}$ Island of promoter regions are shown in Supporting Information Table 1. Additional primer set spanning $32 \mathrm{CpG}$ sites at -149 to +222 bp in relation to transcription start site of the TNFRSF10G cDNA clone NM_003841, and the sequence common to both methylated and unmethylated templates was used for cloning and sequencing (Supporting Information Table 1). PCR was performed using standard conditions for 30 cycles on primary invasive cancer and 35 cycles for precancerous lesions with annealing temperatures varying between 56 and $62^{\circ} \mathrm{C}$. PCR products were run on $2 \%$ agarose gels and visualized after ethidium bromide staining. All methylationspecific PCR (MSP) experiments were performed in triplicate, and the promoter hypermethylation was considered positive when present in replicate experiments. MSP products were subcloned into pCR2.1TOPO vector (Invitrogen) followed by sequencing of multiple clones using M13 primers.

\section{Drug Treatment}

Cells in culture were treated with $5-\mu \mathrm{M} 5$-Aza2'deoxycytidine (5-aza dC) (Sigma-Aldrich) for 5 days by replacing the medium daily, trichostatin (Sigma-Aldrich) at a final concentration of $200 \mathrm{nM}$ for $24 \mathrm{hr}$ and a combination of both as described (Narayan et al., 2009). Human recombinant TRAIL (Invitrogen) dissolved in distilled water, Actinomycin D (Invitrogen) dissolved in DMSO, Cisplatin (LKT laboratories) dissolved in $N, N$ dimethylformamide were used at the indicated concentrations and exposure times. By testing several concentrations on CC cell lines, we found 500 $\mathrm{ng} / \mathrm{ml}$ each of TRAIL and Actinomycin D as optimal for detecting cell death. We determined ICD50 value of Cisplatin by testing concentrations from 0.5 to $50 \mu \mathrm{g} / \mathrm{ml}$, which varied from 2.5 to 16.0 $\mu \mathrm{g} / \mathrm{ml}$ in cell lines used in this study, except SW756 that was resistant even at $50 \mu \mathrm{g} / \mathrm{ml}$ (Supporting Information Fig. 1). Based on this, we used 3$\mu \mathrm{g} / \mathrm{ml}$ final concentration of Cisplatin.

\section{Reverse Transcription Polymerase Chain Reaction (RT-PCR) and Western Blot Analyses}

Total RNA from normal cervical squamous epithelium (three from commercial sources and five from hysterectomy specimens), tumor tissues, and cell lines was reverse transcribed as described elsewhere (Narayan et al., 2003a). Relative quantitation of expression of NKX3-1 (Assay ID Hs00171834) and Human GAPDH as endogenous control (FAM/MGB Probe) genes was performed in triplicate experiments using TaqMan Gene Expression Assay using the Applied Biosystems 7500 Fast Real-Time PCR system (Foster City, CA). Semiquantitative expression of TNFRSF10A, TNFRSF10B, TNFRSF10G, TNFRSF10D, and NKX3-1 was performed in duplicate RT-PGR 
experiments using the primers shown in Supporting Information Table 1 and standard thermal cycle conditions. Immunoblot analysis was performed by standard methods using the antibodies for DR4, DR5, DcR1 and DcR2 (Thermo Scientific, Pierce), CASP8, CASP9, CASP10 (Cell Signalling Technology), and CASP3 (BD Transduction Laboratories). Secondary antibody conjugated with horseradish peroxidase was obtained from Santa-Cruz Technology. Blots were reprobed with $\beta$-actin as control. Detection was performed by ECL-Western Lightning Chemiluminescence reagent (Amersham Pharmacia).

\section{Mutation Analysis}

Sanger sequencing was performed to identify mutations on DNA amplified using the two sets of primers spanning exons 1 and 2 of $N K X 3-1$ gene (Supporting Information Table 1).

\section{Cell Survival and Apoptosis Analysis}

Cell viability and cytotoxicity against TRAIL and other drugs was assessed by standard 3-(4,5dimethylthiazol-2-yl)-2,5-diphenyltetrazolium bromide (MTT; Invitrogen) colorimetric assay. Briefly, 5,000 cells were seeded in 96-well-cell culture plate, grown overnight at $37^{\circ} \mathrm{C}$ in $5 \% \mathrm{CO}_{2}$, treated with indicated concentrations of drugs for $24 \mathrm{hr}$. After incubation of cells in $5 \mathrm{mg} / \mathrm{ml}$ of MTT in phosphate buffer saline for $3.5 \mathrm{hr}$, the dye was dissolved in $150 \mu \mathrm{l}$ of MTT solvent and the optical density of solubilized formazan was assessed using microplate reader (Bio'Tek Quant). All treatments were performed in four replicate wells and repeated three times.

To measure apoptosis, we used Pacific Blue ${ }^{\text {TM }}$ Annexin VSYTOX® AADvanced ${ }^{\mathrm{TM}}$ Apoptosis Kit (Invitrogen). Briefly, one million cells were seeded in 6-well-tissue culture plates, grown overnight at $37^{\circ} \mathrm{C}$ at $5 \% \mathrm{CO}_{2}$ incubator, replaced with new culture medium and added the indicated concentration of drugs. Cells collected after 16 or $24 \mathrm{hr}$ of incubation were analyzed by LSR II flow cytometry (BD Biosciences) using 405 and $488 \mathrm{~nm}$ excitation and 455 and $647 \mathrm{~nm}$ emission. A total of 20,000 events were analyzed using Flowjo software (Tree Star, OR) in duplicate experiments.

\section{Statistical Analysis}

ANOVA and $t$-test statistics were calculated using the GraphPad Prism software (LaJolla, CA).

\section{RESULTS}

\section{Identification of 8p I 2-21.3 Minimal Deleted Region and Candidate Downregulated Genes in CC}

SNP array analysis of 71 primary tumors and 9 cell lines identified copy number alterations (CNA) on chromosome 8 in $38(47.5 \%)$ cases, losses in $30(37.5 \%)$, and gains in $23(28.8 \%)$ cases. Gains were frequently seen on $8 \mathrm{q}$ and the losses on 8 p. Amplification at $8 \mathrm{q} 24.12$ was seen in a single case. Analysis of the dataset with losses on chromosome 8 showed monosomy in three cases and partial deletion in 27 cases (Fig. 1A). Among the tumors that exhibited partial deletions, losses on $8 p$ were only seen in $16(53.3 \%)$ cases, on both chromosomal arms in $10(33.3 \%)$ cases, and only on $8 \mathrm{q}$ in one case. Overall, $8 \mathrm{p}$ was affected by deletion in 29 of $30(96.7 \%)$ of all the deleted cases, suggesting $8 \mathrm{p}$ is the frequent target of deletion in CC. To identify common minimal deleted region (MDR) on $8 \mathrm{p}$, we examined the SNP data for smaller regions of loss. Notably, we found 2 cases (T-117 and T-194) defining the MDR on 8p. The MDR was involved in all cases with $8 \mathrm{p}$ deletions, except 3 (Fig. 1A), which spans an $8.4 \mathrm{Mb}$ region between 22,941 and $31,338 \mathrm{~kb}$ genomic interval on chromosome $8 \mathrm{p}$. All cell lines that had $8 \mathrm{p}$ deletion by SNP array were also showed decreased copies by FISH using a probe spanning the MDR, except in HeLa cell line (data not shown). HeLa cell line showed most part of chromosome 8 loss except distal $8 \mathrm{q}$ gain, while the FISH did not reveal relative decrease of $8 \mathrm{p} 21$ region compared to control centromere probe.

Based on these results, we hypothesized that this decreased $8 \mathrm{p}$ genomic dosage results in loss of function of one or more genes relevant to $\mathrm{CC}$ tumorigenesis. The 8p12-21.3 MDR contains 92 probe sets comprising 53 known genes. To examine the consequence of $8 \mathrm{p}$ deletion on gene expression, we used gene expression profiling of chromosome 8 probe dataset from Affymetrix U133A array on 20 normal (age range, 27-64 year; mean \pm standard deviation $(\mathrm{SD})=46.9 \pm 7.6)$ squamous epithelial samples (including the seven samples used in SNP array), 33 primary tumors (age range $28-70$ year, mean $\pm \mathrm{SD}=48.9 \pm 12.3 ; 22$ of these tumors were also analyzed by SNP array), and 9 cell lines (all analyzed by SNP array). To identify differentially expressed gene signatures of $8 \mathrm{p}$ MDR in $\mathrm{CC}$, we performed supervised analyses using the 92 probe set between tumors and normal, and between $8 \mathrm{p}$ deleted and undeleted tumors using the criteria described in methods. This analysis identified 


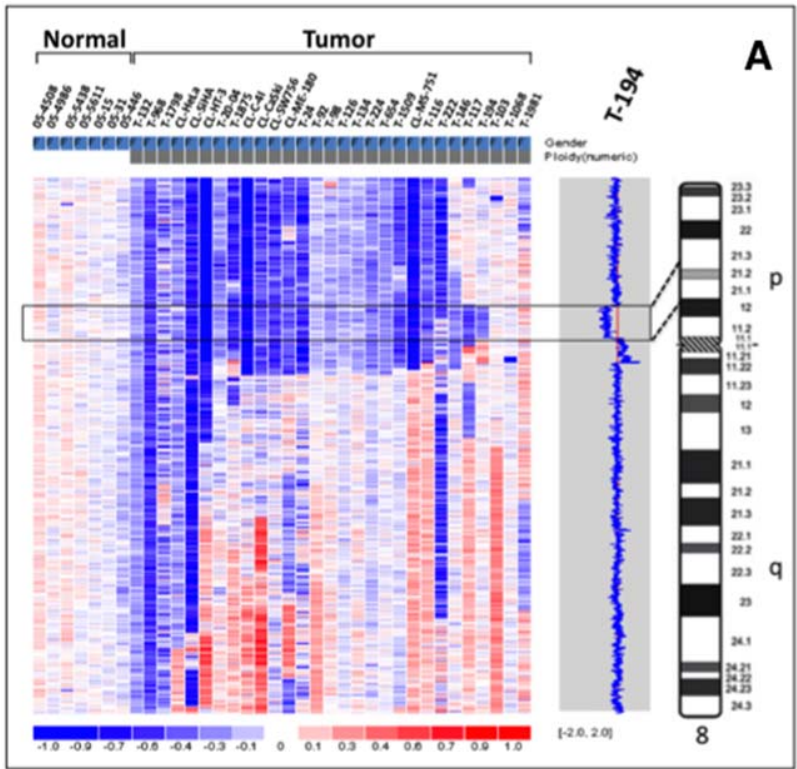

Figure I. Genetic analysis of $8 p$ deletion in CC. (A) Identification of $8 \mathrm{pl} 2-21.3$ minimal deletion by $250 \mathrm{~K}$ Nspl SNP array. Each vertical column represents a sample with genomic regions representing from pter (top) to qter (bottom) on chromosome 8. Prefix "T" indicates primary tumor; "CL" indicates cell line. The blue-red scale bar $(-\mathrm{I}$ to $+I)$ at the bottom represents the copy number changes relative to mean across the samples. The intensities of blue and red indicate relative decrease and increase in copy numbers, respectively. G-banded ideogram of chromosome 8 is shown on the extreme right. All tumors that exhibited chromosome 8 losses are shown from largest to smallest region of deletion. Inferred copy number view of T-194 showing 8p

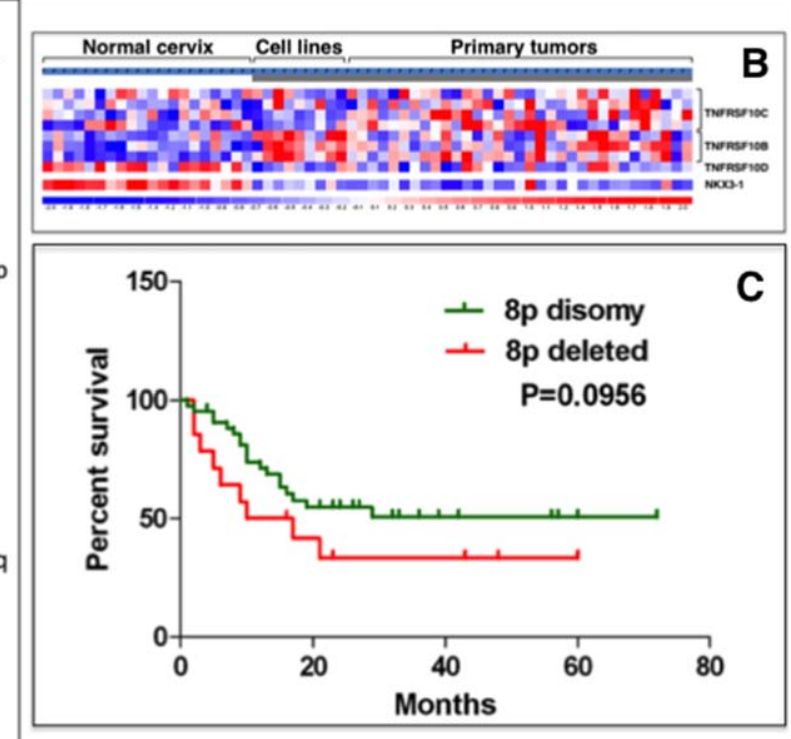

minimal deletion from normal $(2 \mathrm{~N})$ (red line) is shown on right. (B) Gene expression using UI33A array and analysis of genes mapped to MDR in CC cell lines and primary tumors. Significantly differentially expressed genes were identified by comparing normal, cell lines and primary tumor groups. In the matrix, each row represents the gene expression relative to group mean and each column represents a sample. The genes are shown on right. The scale bar $(-2$ to +2$)$ on the bottom represents the level of expression with intensities of blue represents decrease and red for increase in expression. (C) Kaplan-Meier curves showing survival differences between $8 p$ deleted and undeleted cases.

high frequency of tumors $(29 / 42,69 \%)$ showed downregulated expression of TNFRSF10D compared to normal (Fig. 1B). Therefore, this integrative analysis did not identify a candidate target downregulated gene as a consequence of $8 \mathrm{p}$ deletion.

To further examine the significance of $8 \mathrm{p}$ deletion, we evaluated its association with clinical parameters such as age, stage, and size of the tumor, treatment outcome, and HPV type by univariate analyses. $8 \mathrm{p}$ deletions were found to be significantly associated with tumor size $(10 \%$ cases in $<5 \mathrm{~cm}$ vs. $36 \%$ in $>6 \mathrm{~cm} ; P=0.04)$, outcome of the treatment $(18.2 \%$ patients were alive or with complete remission vs. $39.4 \%$ of patients died of disease between 1 and 72 month follow up), and multiple HPV infections (63.6\% in multiple HPV infections vs. $28.9 \%$ in HPV16 alone cases). Although not statistically significant, KaplanMeier survival analysis showed a 2-fold risk of death in patients carrying $8 \mathrm{p}$ deletions (Fig. 1G). These findings thus suggest that $8 \mathrm{p}$ deletion may serve as a predictor of clinical outcome to conventional therapies. 


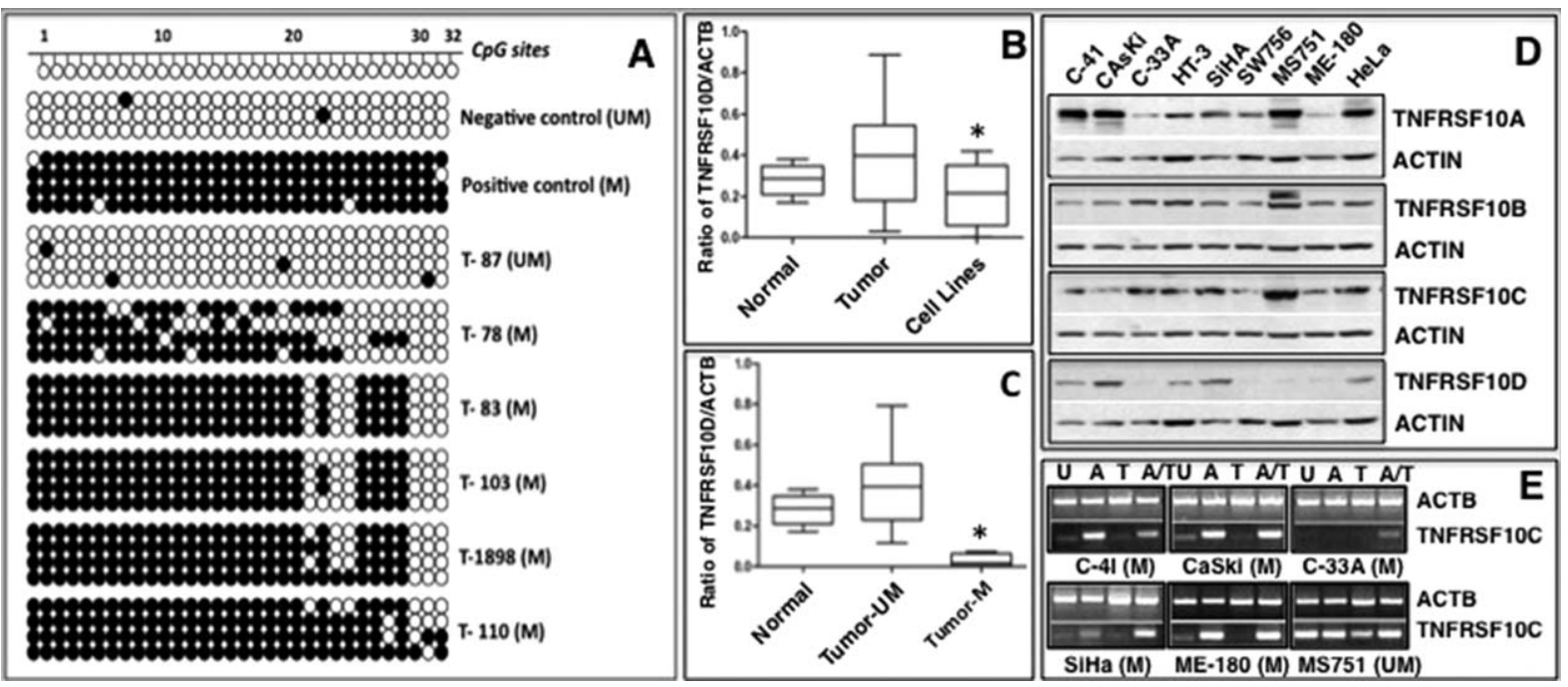

Figure 2. Analysis of TNFRSFIOC methylation and expression in CC. (A) Bisulphite MSP cloning and sequencing. U, unmethylated; $M$, methylated. CPG sites examined are numbered sequentially as shown above. Filled circles indicate methylated $C_{p}$ s sites and empty circles indicate unmethylated $C_{p} G$ s. Each row represents a clone and each panel represents a tumor or control. (B) and (C) RT-PCR analysis of TNFRSFIOC and TNFRSFIOD in CC. (B) Box plot showing semiquantitative analysis in normal, primary tumors and CC cell lines. ANOVA, normal versus tumor, not significant; normal versus cell line $P<0.000$, significant. (C) Box plot showing semiquantitative analysis in normal,

\section{Promoter Hypermethylation-Mediated Inactivation of Decoy Receptor Genes at 8pI2-p2I.3 MDR}

TNF receptor superfamily decoy receptor genes are known to be epigenetically inactivated in multiple tumor types (Shivapurkar et al., 2004). As stated above, the 8p MDR contains a cluster of four TNFRSF10 family genes. TNFRSF10C (DcR1) and TNFRSF10D (DcR2) act as potentially antiapoptotic genes since they lack active death domains, whereas TNFRSF10A (DR4) and TNFRSF10B (DR5) act as proapoptotic genes (Gonzalvez and Ashkenazi, 2010). Based on their function, these genes might be potential targets of 8p MDR. To understand their role, we examined the promoter hypermethylation status of these genes in 123 invasive cancers (9 cell lines and 114 primary tumors) by MSP analysis. Promoter methylation was identified in TNFRSF10C and TNFRSF10D, while it was absent in TNFRSF10A and TNFRSF10B. Hypermethylation of TNFRSF10G was found in $56(45.5 \%)$ of the 123 CC cases (cell lines, 88.9\%; primary tumors, 42.1\%; Table 1). The other decoy receptor TNFRSF10D showed promoter hypermethylation in $5.7 \%$ tumors. DNA isolated from 34 normal cer- unmethylated, and methylated tumors. ANOVA, normal versus unmethylated, not significant; normal versus methylated $P<0.0004$, significant. Box plots show median, 25th and 75th percentile, minimum and maximum values. (D) Immunoblot analysis showing expression of TNFRSFIO receptors. Actin was used as a loading control. (E) Effect of drug treatment using inhibitors of methylation and HDAC on gene reactivation by RT-PCR analysis in CC cell lines. Bracketed $M$ and UM indicates methylated and unmethylated cell lines, respectively. ACTB, beta actin; U, untreated; A, 5-Aza-2'-deoxycytidine; T, TSA; A/T, 5-Aza2 -deoxycytidine and TSA.

vical epithelia did not reveal hypermethylation in these promoters. To validate the MSP data, we performed bisulfite sequencing of a region covering 32 CpGs within the $\mathrm{CpG}$ island of TNFRSF10C in one unmethylated and five methylated primary tumors, chosen randomly to represent each of these classes, along with the controls confirmed the MSP results (Fig. 2A).

Of the 9 cell lines studied, 8 were MSP positive for TNFRSF10G without evidence for the presence of an unmethylated allele. These data combined with SNP array (Fig. 1A) suggest that 7 of 8 cell lines with $8 \mathrm{p}$ deletion had methylation of the second allele. The remaining cell line (MS751) that harbor $8 \mathrm{p}$ deletion by SNP array did not show promoter methylation of TNFRSF10C. The C-33A cell line that had disomy for chromosome $8 \mathrm{p}$ by SNP array exhibited both methylated alleles. Therefore, these data supports that TNFRSF10C is one of the targets of chromosome $8 \mathrm{p}$ deletion. Thus, the cell line data suggest that the TNFRSF10G inactivation follow the "two-hit" hypothesis (Knudson and Strong, 1972). However, only 9 of $21(43 \%)$ primary tumors showed simultaneous loss of $8 \mathrm{p}$ and TNFRSF10C promoter methylation. Similarly, $18(36 \%)$ of 50 tumors 
without 8 p deletion also showed promoter methylation. Therefore, no strict association between methylation and $8 \mathrm{p}$ deletion could be established in primary tumors.

Since the TNFRSF10C expression using U133A array was unclear in relation to $8 \mathrm{p}$ deletion, we extended the expression studies using real time RT-PCR to examine if aberrant promoter hypermethylation is associated with transcriptional downregulation. TNFRSF 10C expression on a panel of 20 normal cervical epithelia, 9 CC cell lines, and 25 tumor specimens showed no significant downregulation relative to $\beta$-actin between primary tumors $($ mean $\pm \mathrm{SD}=0.81 \pm 0.33)$ and normal epithelium (mean $\pm \mathrm{SD}=0.94 \pm 0.15)$. However, the expression in the cell lines (mean $\pm \mathrm{SD}=0.36 \pm 0.34$ ) was significantly decreased compared to normal $(P<0.0001)$ and primary tumors $(P=0.0017$; Fig. $2 \mathrm{~B})$. Further analysis to examine the relationship between methylation and gene expression showed significant differences in TNFRSF10C levels between normal and methylated tumors (mean$\pm \mathrm{SD}=0.58 \pm 0.40 ; \quad P<0.0004)$, while no difference between normal and unmethylated tumors (mean $\pm \mathrm{SD}=0.99 \pm 0.11 ; P=0.48$ ) was found. Similarly, the expression levels were also significantly $(P=0.004)$ lower in methylated tumors compared to unmethylated tumors (Fig. 2G). Thus, the RT-PCR data suggest that promoter hypermethylation of TNFRSF10G results in transcriptional downregulation. Furthermore, western blot analysis of cell lines showed all methylated cell lines exhibited decreased protein levels of TNFRSF10C, while the unmetylated cell line (MS751) expressed high levels of protein (Fig 2D). For TNFRSF10D gene, six of the 9 cell lines showed relatively low protein. Two of 3 methylated cell lines and four of 6 unmethylated cell lines showed decreased levels of TNFRSF10D protein (Fig. 2D). Of note, TNFRAF10A showed relative low levels of protein in four of 9 cell lines, while TNFRSF10B showed no detectable decrease. Overall, these data suggest that promoter methylation or other epigenetic mechanisms results in downregulation of TRAIL decoy receptors in the majority of CC cell lines.

To examine the role of DNA hypermethylation and histone modifications in gene silencing, we treated eight methylated and one unmethylated cell lines with 5-aza dC and TSA. RT-PCR analysis of these cells showed various levels of reactivation of TNFRSF10C and with Aza or Aza with TSA treatments, but not with TSA alone, in all methylated cell lines. However, no reactivation was found in unmethylated cell line MS751 (Fig.
2E). These data confirm that the methylation of TNFRSF10C promoter results in downregulated expression and demethylation effectively reactivates the gene expression by reversing the methylation affect.

To examine the prognostic role of TNFRSF10C hypermethylation, we performed a correlative analysis of methylation with clinicopathologic features such as age, tumor stage, and size of the tumor, clinical outcome, and HPV type in primary tumors and found no significant associations (data not shown).

\section{DNA Damaging Agents Cisplatin and Actinomycin D Sensitizes TRAIL Mediated Apoptotic Response in 8p-Deleted CC Cell Lines}

TNF-related apoptosis-inducing ligand or Apo2L (TRAIL) binds to its agonistic receptors triggering apoptosis. TRAIL therapy is evaluated as a promising tool in several cancer clinical trails. However, the efficacy of TRAIL varies among tumors owing to defects in death-inducing signaling complex (DISC) formation. Since $8 p$ deleted CG cases showed adverse outcome to standard therapies, we examined if the efficiency of TRAIL-induction of apoptosis is $8 \mathrm{p}$ deletion dependent. Treatment of TRAIL showed higher apoptotic response in 8p-deleted cell lines ( $\mathrm{SiHa}$, C-4I, CaSki, SW-756, HeLa, ME-180, HT-3, and MS-751) compared to an undeleted cell line (C33A) $(P=0.0002$; Fig. 3A, lane 1). Exposure to antineoplastic DNA-intercalating agents Cisplatin or Actinomycin D significantly increased apoptosis in 8p-deleted cell lines compared to undeleted cell line (Fig. 3A, lanes 2 and 3). To examine whether these drugs enhance the TRAILmediated apoptosis, we tested TRAIL combined with Cisplatin or Actinomycin D, and identified a synergistic effect by both the drugs showing significant increase in 8p-deleted cell lines (Fig. 3A, lanes 4 and 5). Although the differences were less significant by cytotoxicity assays (Supporting Information Fig. 3) these results indicate that $8 p$ deletion effectively enhances lethality of Cisplatin or Actinomycin D combination with TRAIL treatment in CG cells.

\section{Epigenetic Inactivation of Decoy Receptor Expression Effectively Mediates TRAIL-Induced Apoptosis}

Since 8p-deleted CG cells are sensitive to TRAIL-induced apoptosis and TRAIL mediated 

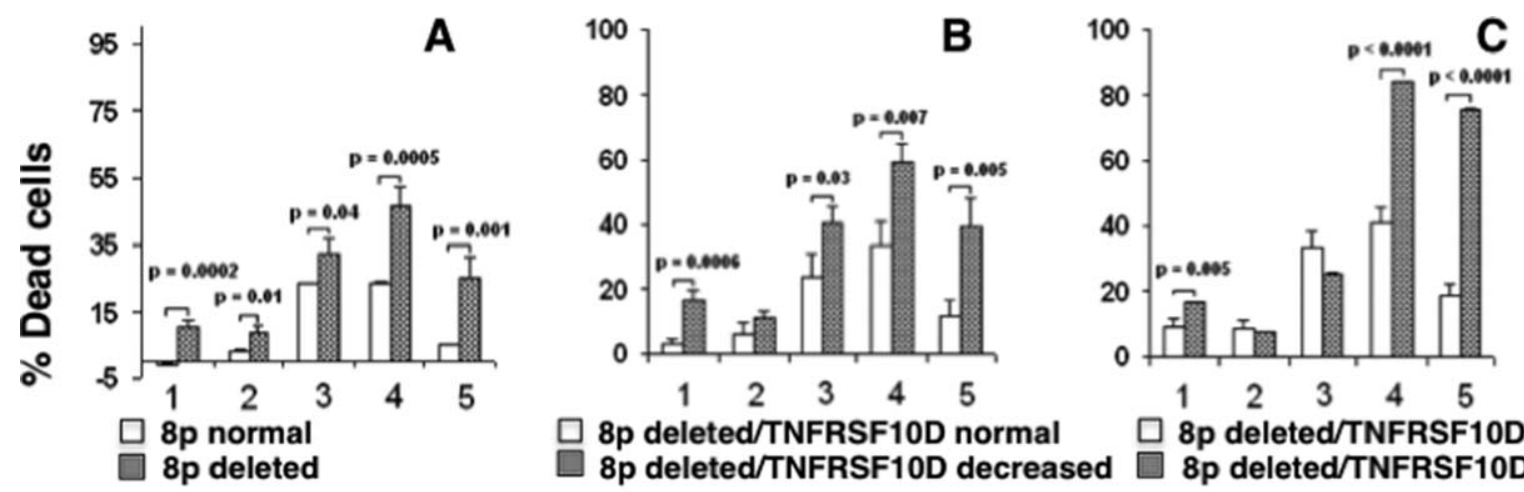

$8 \mathrm{p}$ deleted/TNFRSF10D normal $\square$ 8p deleted/TNFRSF10D-UM $8 \mathrm{p}$ deleted/TNFRSF10D decreased $\square$ 8p deleted/TNFRSF10D-M

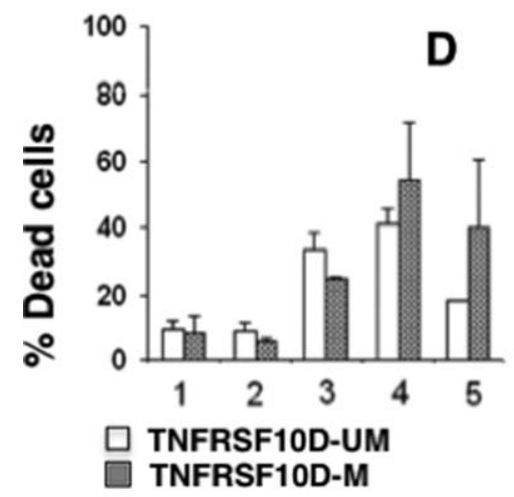

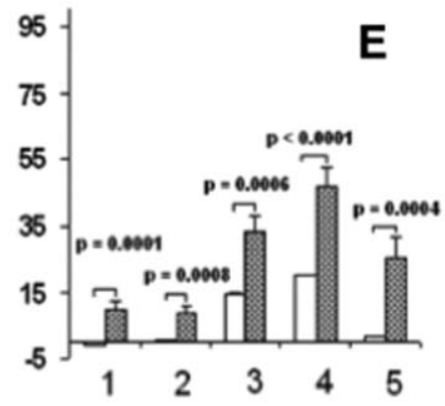

$\square$ 8p deleted/TNFRSF10C-UM

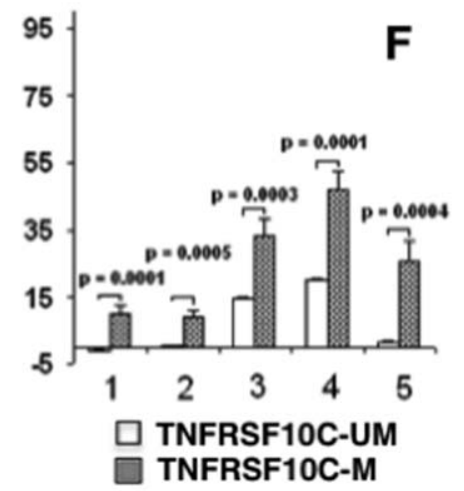

\section{$1=0.5 \mu \mathrm{g} / \mathrm{ml}$ TRAIL \\ $2=3 \mu \mathrm{g} / \mathrm{ml}$ Cisplatin \\ $4=0.5 \mu \mathrm{g} / \mathrm{ml}$ TRAIL $+0.5 \mu \mathrm{g} / \mathrm{ml}$ Actinomycin D}

Figure 3. Decoy receptor inactivation is a positive biomarker in mediating TRAIL-induced apoptosis in CC cell lines. Cells were treated with indicated drugs and concentrations for $2 \mathrm{hr}$. The percent total cell death was measured as sum of Annexin $V$ positive, Annexin V/ SYTOX AADvanced double positive and SYTOX AADvanced positive cells using Annexin V/SYTOX AADvanced kit (Invitrogen) and FACS analysis. (A) Comparison of cell death between with or without 8pdeleted CC cell lines. (B) Analysis of cell death in relation to TNFRSFIOD expression levels in 8 p deleted CC cell lines. (C) Analysis

apoptotic response depend on the balance of expression of its death receptor (DR4 and DR5) and antagonistic decoy receptor (DcR1 and DcR2) genes, we next want to elucidate the role of decoy receptor inactivation in drug response. Of the 8 cell lines that showed $8 \mathrm{p}$ deletion, four $(\mathrm{SiHa}, \mathrm{C}-$ 4I, SW-756, MS-751) had normal levels of TNFRSF10D expression, while the remaining four (CaSki, HeLa, ME-180, HT-3) showed decreased expression (Figs. 1B and 2D). We found that treatment of TRAIL alone or combined with Cisplatin or Actinomycin D showed a significantly high apoptosis and reduced cellular viability in cell lines with decreased expression of TNFRSF10D compared to the cell lines that expressed normal levels of TNFRSF10D (Fig. 3B and Supporting Information Fig. 3B). The cell line (ME-180) that showed $8 \mathrm{p}$ deletion and TNFRSF10D methylation also showed high-apoptotic response and sensitivity to of cell death in relation to TNFRSFIOD methylation in 8p-deleted CC cell lines. (D) Analysis of cell death in relation to TNFRSFIOD methylation independent of $8 p$ deletion in CC cell lines. (E) Analysis of cell death in relation to TNFRSFIOC methylation and expression in 8pdeleted CC cell lines. (F) Analysis of cell death in relation to TNFRSFIOC methylation and expression independent of 8p deletion in $\mathrm{CC}$ cell lines. Statistically significant differences are indicated at the top for each group.

cell death as compared to $8 \mathrm{p}$ deleted but TNFRSF10D unmethylated cell lines (Fig. 3C, Supporting Information Figs. 3C and 4). The cell lines (C-33A and ME-180) that exhibited TNFRSF10D methylation showed a similar sensitivity to cell death irrespective of $8 \mathrm{p}$ deletion status (Fig. 3D and Supporting Information Fig. 3D). These data, thus, suggest that TRAIL-induced cell death response is dependent on either the status of methylation and/or the levels of expression of TNFRSF10D. This affect was synergistic in the presence of Cisplatin or Actinomycin D (Fig 3, Supporting Information Fig 3).

We then examined changes in TNFRSF10C gene in response to these drugs. When compared $\mathrm{CC}$ cell lines with the combination of $8 \mathrm{p}$ deletion, promoter methylation with down regulated expression (SiHa, C-4I, CaSki, SW-756, HeLa, ME-180, and HT-3) and an unmethylated with 


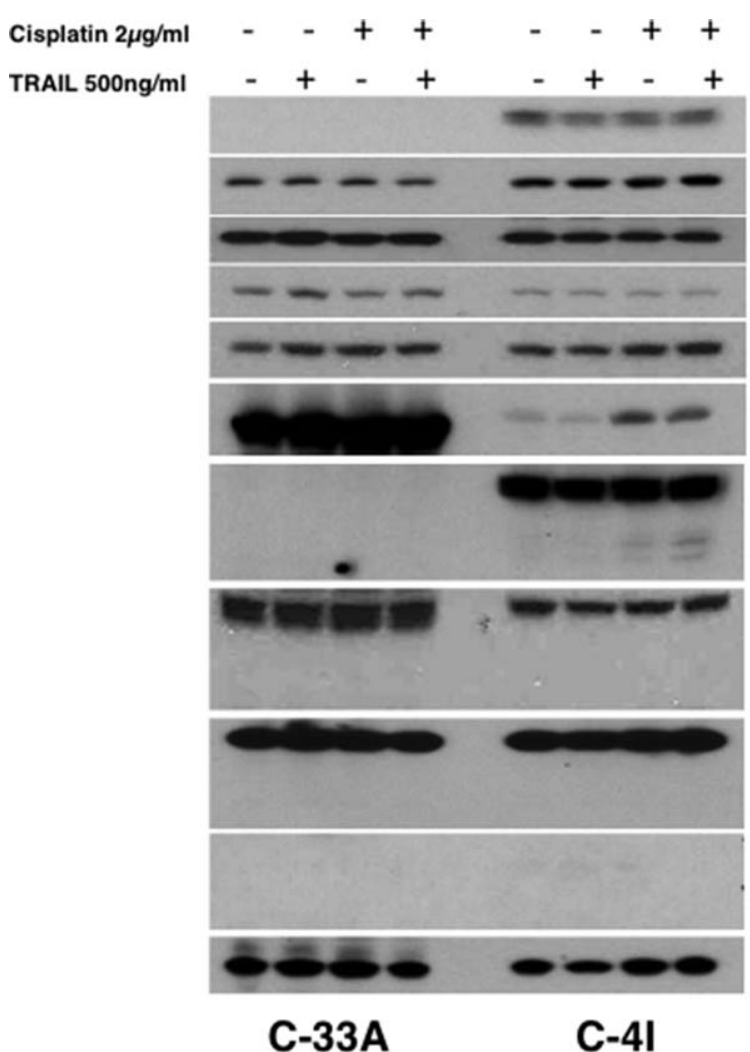

Figure 4. Activation of extrinsic apoptotic pathway after TRAILCisplatin combination treatment in CC cell lines depends on the methylation/inactivation status of TRAIL receptors. Western blot analysis showing Caspase-8, caspase-9, caspase-3, caspase-10, DR4, DR5, DcRI, DcR-2, and TP53 expression after treatment with Cisplatin, TRAIL, or combination of both. Cleaved fragments with molecular size

normal levels of expression (MS-751), the data further revealed that the unmethylated cell line exhibits resistance to cell death to TRAIL- and other combination drug treatments. This effect was significantly synergistic to TRAIL combined with Cisplatin or Actinomycin D (Figs. 3E and 3F, Suppl Figs. 3E, 3F, and 4).

Therefore, these in vitro experiments establish that the tumor cells carrying a combination of methylated alleles and/or downregulated transcription of one or both decoy receptors elicit efficient antitumor effects against TRAIL-combination treatments.

\section{Epigenetic Inactivation of Decoy Receptors Facilitates Efficient Activation of Extrinsic Apoptotic Pathway}

Since we observed enhanced cell death response to TRAIL-combination drug treatments in cell lines expressing low levels of DcR1 and DcR2, as well as apoptotic resistance in cell line

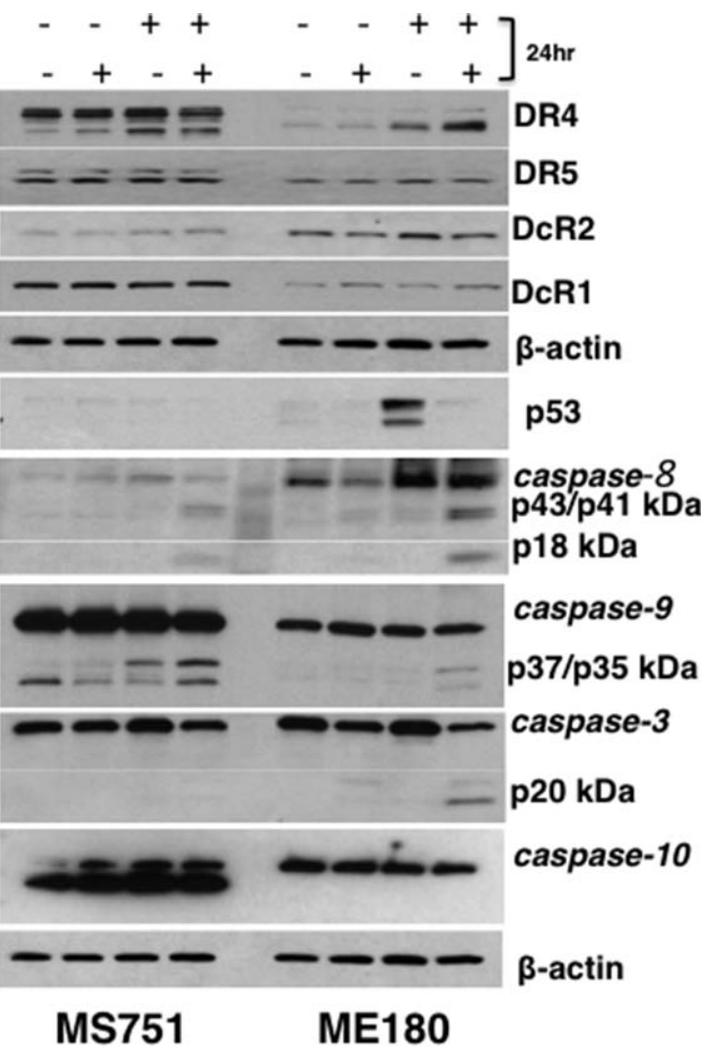

markers are shown on the right in $\mathrm{kDa}$. These results confirmed on at least 3 independent experiments. Right panel: Western blot analysis showing activation of caspases- $8,-9$, and -3 in MEI 80 cell line; caspase- 8 and -9 activation in MS75I cell line. Left panel showing lack of caspase activation in C-4I (only caspase-8 activated) and C-33A cell lines.

expressing low level of death receptor DR4, we wanted to examine the relation between receptor expression, and caspase activation to TRAILCisplatin treatments. To test this, we chose four cell lines with variations in decoy receptor methylation/downregulated expression exhibiting extreme sensitivity or resistance to TRAIL/Cisplatin treatment. ME-180 cells carrying promoter hypermethylation/inactivation of DcR 1 and 2 as well as normal expression of death receptors was the cell line that exhibited maximum response to TRAIL, Cisplatin, or Actinomycin treatments. Upon treatment of ME-180 cells with TRAIL, Cisplatin, or combination of both resulted in activation of initiator CASP8. However, other initiator caspase CASP10 showed no evidence of activation (Fig. 4). The effector caspase CASP3 is also highly activated after treatment with TRAIL combined with Cisplatin (Fig. 4). Of note, in addition CASP9 that plays a role in mitochondrial apoptotic pathway, is also activated. These data thus suggest active apoptotic pathway in this cell line. In 
addition, proapoptotic receptor DR4 levels were elevated after treatment with Cisplatin alone or in combination with TRAIL, while DR5 levels remain unchanged. Thus the TRAIL + Cisplatin treatment activates DR4 leading to active caspase pathway in this cell line (Fig. 4). Thus, we assume that the inactivated decoy receptors in ME-180 facilitate high sensitivity to cell death via extrinsic apoptotic signaling. A second cell line C-4I with promoter methylation associated inactivation of DcR1 that also exhibited high rate of cell death after the drug treatment, showed activation of only initiator caspase, 8 , but no detectable activation of downstream caspases CASP3 or 9 was found. Treatment did not result in any detectable changes in DR4 or DR5 levels in this cell line. Therefore, high sensitivity to TRAIL/Cisplatin resulting in activated caspase pathway in ME-180 is due to concomitant inactivation of both decoy receptors. However, the second cell line C4-I that also exhibits high sensitivity to TRAIL did not effectively activate downstream caspases. Since only one decoy receptor is inactivated in this cell line, other alternative pathways such as mitochondrial pathway may also play role in sensitizing the these cells. Of note, TP53 is activated on treatment with Cisplatin or Cisplatin combined with TRAIL in C-4I supporting TP53-dependent pathway may be active.

The MS751 cell line carrying unmethylated promoters and detectable levels expression of DcR genes exhibited highest resistance to TRAIL/Cisplatin. Treatment of this cell line with TRAIL/ Cisplatin results in activation of caspases 8 and 9 , but no evidence of CASP3 activation was found. Thus, this finding suggests defective activation of caspase pathway in this cell line (Fig. 4). A second cell line C-33A despite downregulated expression of both DcR genes was resistant to TRAIL/Cisplatin treatment (Fig. 4). No evidence of caspase activation was seen in this cell line after treatment. This cell line carries TP53 mutation and exhibits complete lack of DR4 expression. Thus, the apoptotic resistance in this cell line is due to DR4 absence and possibly TP53 also plays a role in resistance, which may override the advantage conferred by decoy receptor inactivation promoting cell death response. Thus, these data suggest that decoy receptor inactivation promotes cell death response to TRAIL-Cisplatin treatment, in the presence of active death receptors, by activating the caspases that play role in extrinsic apoptotic pathway.

\section{TNFRSFIOC Promoter Hypermethylation Occurs at an Early Stage in CC Development}

To identify the role of promoter hypermethylation of TNFRSF10G gene in CG progression, we studied methylation status in DNA obtained from 141 cytological smears diagnosed as normal $(N=34)$, ASC-US $(N=22)$, LSIL $(N=43)$, or HSIL $(N=42)$. We found no evidence of methylation in DNA from cytologic smears diagnosed normal. In contrast, $5(22.7 \%)$ of 22 cases diagnosed as ASC-US, $8(18.6 \%)$ of 43 LSILs and 5 (11.9\%) of 42 HSILs showed promoter hypermethylation by MSP analysis (Table 1). Thus, these data provide evidence that promoter hypermethylation of TNFRSF 10G initiate at an early developmental stage during CC tumorigenesis.

\section{DISCUSSION}

Chromosome arm 8p suffers frequent deletions in many human epithelial and hematologic malignancies (http://cgap.nci.nih.gov/Chromosomes/ RecurrentAberrations). Here, we show that the 8p12-21.3 as the MDR in CG and the deletion prognosticates a 2-fold increased risk of death. Therefore, it is likely that one or more proliferation-regulated genes are situated in the deleted region and their inactivation plays a role in CC. The MDR at 8p12-21.3 contains a number of genes known to play a role in human cancer. In this study, we identified TNFRSF10G and TNFRSF10D spanning the MDR as target inactivated genes by genetic and/or epigenetic mechanisms.

The proteins encoding TNFRSF10C and TNFRSF10D belongs to the TNF-receptor family containing an extracellular TRAIL-binding domain and a transmembrane domain, but lacks the cytoplasmic death domain. Thus, these receptors are not capable of inducing apoptosis. Although DcR1 and DcR2 express on cell surface lack functional intracellular death domain, they are fully functional. DcR1 and DcR2 inhibit TRAIL-induced apoptosis by competing with proapoptotic TRAIL receptors DR4 and DR5 in binding TRAIL, thus act as antagonistic molecules that protect cells from TRAIL-induced apoptosis by preventing the assembly of death-inducing signaling complex (DISC) (Sheridan et al., 1997; Micheau and Merino, 2004). TNFRSF10C and TNFRSF10D genes have been shown to be inactivated by promoter hypermethylation in multiple tumor types (van Noesel et al., 2002; Shivapurkar et al., 2004; Yang et al., 2007; Hornstein et al., 
2008; Braga Lda et al., 2012; Mahapatra et al., 2012). Here, we identified functional inactivation of TNFRSF10G and TNFRSF10D in a high proportion of $\mathrm{CG}$ by promoter hypermethylation or downregulated expression as shown by the previous reports (Shivapurkar et al., 2004, 2007). Thus, the decoy receptor inactivation identified in this study suggests that TRAIL-induced apoptosis may be an effective treatment option in patients exhibiting inactive TNFRSF10G and/or TNFRSF10D combined with the conventional chemoradiotherapy in CC. Although TRAILinduced apoptosis is a promising approach in cancer therapy, not all tumors are sensitive to TRAIL. The mechanisms involved in TRAIL resistance are not fully understood. One postulated mechanisms have been that overexpression of DcR1 or DcR2 protect tumor cells from TRAIL-induced apoptosis (Sheridan et al., 1997). In this study, we have demonstrated that inactive $D c R 1$ and $D c R 2$ genes can enhance TRAIL-mediated apoptosis in CC. Our studies further suggest that combination of chemotherapeutic agents such as Actinomycin D or Cisplatin with TRAIL are highly effective in achieving increased TRAIL-induced apoptosis in tumor cell lines with $8 \mathrm{p}$ deletion or inactivated DcR 1 and DcR2 genes. Thus, our data demonstrate that patients carrying $8 \mathrm{p}$ deletion and/or decreased expression of decoy receptors in their tumor cells may benefit from combination chemotherapeutic drugs with recombinant TRAIL. Many previous studies focused on mechanisms of TRAIL resistance and the mechanisms of tumor sensitivity are largely ignored. This study demonstrates that at least one of the mechanisms of sensitivity is decoy receptor downregulation. However, a better understanding of biologic mechanisms underlying tumor response with logical combination of drugs in $\mathrm{CG}$ is needed to identify and enrich responsive patient selection.

Combination therapies can affect more than one death pathways. For example, expression of TRAIL receptors and their decoy receptors can be induced by wild-type TP53 and other transcription factors upon treatment with DNA damaging agents (Takimoto and El-Deiry, 2000; Liu et al., 2005). Thus, the DNA damaging agents such as chemotherapy and radiation can be more effective in combination with TRAIL and synergistic effect has been demonstrated in a number of tumor types (Mahalingam et al., 2009).

Since TRAIL receptors are TP53-regulated DNA damage-inducible genes, mutations in TP53 may impair TRAIL-induced apoptotic activity
(Ruiz de Almodovar et al., 2004; Liu et al., 2005). The majority of CG are HPV 16 positive, where high-risk HPV E6 protein causes TP53 degradation, and hence nonfunctional. In this scenario, it is expected that the HPV16-positive cervical tumors may have impact on TRAIL therapy response. The present data in relation to HPV infection and TP53-mutated CG cell lines showed that TP53-mutated tumors exhibited slightly higher resistance to TRAIL, but the difference were not statistically significant between HPV positive and negative cell lines (Supporting Information Figs. 5A and 6A). This is consistent with nonfunctional TP53 in mutated and HPV-negative cell lines, while HPV-positive tumors may still express low levels of functional TP53 and exhibit slightly higher response to TRAIL. Our data suggest that HPV-negative tumors carrying TP53 mutations may be more resistant to TRAIL therapy. Whether the type of high-risk HPV determines response to TRAIL and combination with DNA damaging agents is not known. Our in vitro data showed that the tumors carrying HPV 16 and HPV18/45 exhibited significantly higher resistance to TRAIL and combination with Actinomycin D or Cisplatin compared to cell lines carrying HPV 18, 16/18, 39/68 (Supporting Information Figs. 5B$5 \mathrm{~F}$ and $6 \mathrm{~B}-6 \mathrm{~F}$ ). However, these observations and the mechanisms of TRAIL resistance in relation to HPV infection remain to be validated further in a larger series of cell lines.

Synergy between DNA damaging agents and TRAIL has been well established in a variety of tumors (Morizot et al., 2011). Our present in vitro studies provide evidence that decoy receptor inactivation potentially enhances antitumor efficacy of TRAIL by activating the extrinsic death pathway. However, the relative contribution of signals that trigger apoptosis by TRAIL sensitization depends on large variety of events ranging from DISC formation to more distal events. CFLAR, regulator of apoptosis structurally similar to caspase- 8 and a critical molecular at DISC formation, is one such gene. CFLAR downregulation of either full length or short variants have been shown to either induce or reduce TRAIL-induced apoptosis (Irmler et al., 1997). We have previously reported that the chromosomal region that maps CFLAR was deleted in both precancerous and cancerous lesions of cervix, and its downregulation in cell lines (Narayan et al., 2003b). Although TRAIL-Cisplatin inducted caspase activation correlate with inactivation of decoy receptor genes, the activation of caspases is highly variable. One mechanism, as has 
been shown in C-33A cell line, is that lack of DR4 expression fails to activate the extrinsic pathway. Downstream pathway gens such as CFLAR remains to be assessed for their role in activation of caspases. Thus, further analysis of downstream pathway genes may clarify the mechanisms of TRAIL sensitivity and/or resistance in CC.

In this study, we also identified TNFRSF10G promoter methylation in all grades of precancerous lesions (Table 1). The potential of individual cervical precancers to progress to higher grades is currently unpredictable (Syrjanen, 1996; McCredie et al., 2008; Schiffman and Rodriguez, 2008). Whether the TNFRSF10C methylation and the associated inactivation will serve as a potential signature in defining risk of progression or even serve as biomarker of treatment of precancerous lesions remains to be understood and warrants further studies.

TNFRSF10C promoter methylation was found in $23 \%$ of specimens with ASC-US diagnosis. A cytological diagnosis of ASC-US is reported in around $5 \%$ of women undergoing Pap screening but the clinical relevance of ASC-US is largely unclear. Follow-up studies of patients with this unequivocal diagnosis show histologically highgrade disease (CIN) lesions or even invasive cancer in a proportion of cases (The ASC-US-LSIL Triage Study (ALTS) Group, 2003). However, no optimal strategy for ASC-US triage to identify high-grade disease that requires follow-up and treatment is available in limiting the number of women who receive unnecessary procedures. Since we found TNFRSF 10 C methylation in $23 \%$ patients with the diagnosis of ASC-US, whether these patients represent already underlying highgrade disease remains to be determined.

In conclusion, our present results clearly demonstrate that patients with detectable 8p12-21.3 deletion harboring multiple tumor suppressor genes are at risk for disease progression and poor outcome. We identified inactivation of TNFRSF10C and TNFRSF10D in majority of CC cases. We also demonstrated that cell lines with inactivated DcR1 and DcR2 showed enhanced TRAIL-induced cell death in combination drug therapy by efficient activation of extrinsic apoptotic pathway. Therefore, it is anticipated that patients that lack expression of decoy receptors may display enhanced response to TRAILcombination therapies. Strategies that downmodulate decoy receptors might also be a viable approach for an affective TRAIL therapy in CC patients.

\section{REFERENCES}

Beroukhim R, Brunet JP, Di Napoli A, Mertz KD, Seeley A, Pires MM, Linhart D, Worrell RA, Moch H, Rubin MA, Sellers WR, Meyerson M, Linehan WM, Kaelin WG, Jr., Signoretti S. 2009. Patterns of gene expression and copy-number alterations in von-hippel lindau disease-associated and sporadic clear cell carcinoma of the kidney. Cancer Res 69:4674-4681.

Bhattacharya N, Singh RK, Mondal S, Roy A, Mondal R, Roychowdhury S, Panda CK. 2004. Analysis of molecular alterations in chromosome 8 associated with the development of uterine cervical carcinoma of Indian patients. Gynecol Oncol 95:352-362.

Braga Lda C, Alvares da Silva Ramos AP, Traiman P, Silva LM, Lopes da Silva-Filho A. 2012. TRAIL-R3-related apoptosis: epigenetic and expression analyses in women with ovarian neoplasia. Gynecol Oncol 126:268-273.

Chang BL, Liu W, Sun J, Dimitrov L, Li T, Turner AR, Zheng SL, Isaacs WB, Xu J. 2007. Integration of somatic deletion analysis of prostate cancers and germline linkage analysis of prostate cancer families reveals two small consensus regions for prostate cancer genes at 8p. Cancer Res 67:4098-4103.

Chua YL, Ito Y, Pole JC, Newman S, Chin SF, Stein RC, Ellis IO, Caldas C, O'Hare MJ, Murrell A, Edwards PA. 2009. The NRG1 gene is frequently silenced by methylation in breast cancers and is a strong candidate for the $8 \mathrm{p}$ tumour suppressor gene. Oncogene 28:4041-4052.

Gonzalvez F, Ashkenazi A. 2010. New insights into apoptosis signaling by Apo2L/TRAIL. Oncogene 29:4752-4765.

Hampton GM, Larson AA, Baergen RN, Sommers RL, Kern S, Cavenee WK. 1996. Simultaneous assessment of loss of heterozygosity at multiple microsatellite loci using semi-automated fluorescence-based detection: subregional mapping of chromosome 4 in cervical carcinoma. Proc Natl Acad Sci U S A 93: 6704-6709.

Harris CP, Lu XY, Narayan G, Singh B, Murty VV, Rao PH. 2003. Comprehensive molecular cytogenetic characterization of cervical cancer cell lines. Genes Chromosomes Cancer 36:233241.

Hornstein M, Hoffmann MJ, Alexa A, Yamanaka M, Muller M, Jung V, Rahnenfuhrer J, Schulz WA. 2008. Protein phosphatase and TRAIL receptor genes as new candidate tumor genes on chromosome $8 \mathrm{p}$ in prostate cancer. Cancer Genomics Proteomics 5:123-136.

Irmler M, Thome M, Hahne M, Schneider P, Hofmann K, Steiner V, Bodmer JL, Schroter M, Burns K, Mattmann C, Rimoldi D, French LE, Tschopp J. 1997. Inhibition of death receptor signals by cellular FLIP. Nature 388:190-195.

Kloth JN, Oosting J, van Wezel T, Szuhai K, Knijnenburg J, Gorter A, Kenter GG, Fleuren GJ, Jordanova ES. 2007. Combined array-comparative genomic hybridization and singlenucleotide polymorphism-loss of heterozygosity analysis reveals complex genetic alterations in cervical cancer. BMC Genomics $8: 53$

Knudson AGJ, Strong LC. 1972. Mutation and cancer: neuroblastoma and pheochromocytoma. Am J Hum Genet 24:514-532.

Li C, Wong WH. 2001. Model-based analysis of oligonucleotide arrays: expression index computation and outlier detection. Proc Natl Acad Sci USA 98:31-36.

Lin M, Wei LJ, Sellers WR, Lieberfarb M, Wong WH, Li C. 2004. dChipSNP: significance curve and clustering of SNParray-based loss-of-heterozygosity data. Bioinformatics 20:12331240 .

Liu X, Yue P, Khuri FR, Sun SY. 2005. Decoy receptor 2 (DcR2) is a p53 target gene and regulates chemosensitivity. Cancer Res 65:9169-9175.

Macartney-Coxson DP, Hood KA, Shi HJ, Ward T, Wiles A, O'Connor R, Hall DA, Lea RA, Royds JA, Stubbs RS, Rooker S. 2008. Metastatic susceptibility locus, an $8 p$ hot-spot for tumour progression disrupted in colorectal liver metastases: 13 candidate genes examined at the DNA, mRNA and protein level. BMC Cancer 8:187

Mahalingam D, Szegezdi E, Keane M, Jong S, Samali A. 2009. TRAIL receptor signalling and modulation: Are we on the right TRAIL? Cancer Treat Rev 35:280-288.

Mahapatra S, Klee EW, Young CY, Sun Z, Jimenez RE, Klee GG, Tindall DJ, Donkena KV. 2012. Global methylation profiling for risk prediction of prostate cancer. Clin Cancer Res 18:28822895. 
McCredie MR, Sharples KJ, Paul C, Baranyai J, Medley G, Jones RW, Skegg DC. 2008. Natural history of cervical neoplasia and risk of invasive cancer in women with cervical intraepithelial neoplasia 3: a retrospective cohort study. Lancet Oncol 9:425434.

Micheau O, Merino D. 2004. Controlling TRAIL-mediated caspase-3 activation. Leukemia 18:1578-1580.

Midorikawa Y, Yamamoto S, Tsuji S, Kamimura N, Ishikawa S, Igarashi H, Makuuchi M, Kokudo N, Sugimura H, Aburatani H. 2009. Allelic imbalances and homozygous deletion on 8p23.2 for stepwise progression of hepatocarcinogenesis. Hepatology 49:513-522.

Mitra AB, Murty VV, Li RG, Pratap M, Luthra UK, Chaganti RS. 1994. Allelotype analysis of cervical carcinoma. Cancer Res 54: 4481-4487.

Morizot A, Merino D, Lalaoui N, Jacquemin G, Granci V, Iessi E, Lanneau D, Bouyer F, Solary E, Chauffert B, Saas P, Garrido C, Micheau O. 2011. Chemotherapy overcomes TRAIL-R4mediated TRAIL resistance at the DISC level. Cell Death Differ 18:700-711.

Narayan G, Arias-Pulido H, Koul S, Vargas H, Zhang FF, Villella J, Schneider A, Terry MB, Mansukhani M, Murty VV. 2003a. Frequent promoter methylation of CDH1, DAPK, RARB, and HIC1 genes in carcinoma of cervix uteri: its relationship to clinical outcome. Mol Cancer 2:24

Narayan G, Pulido HA, Koul S, Lu XY, Harris CP, Yeh YA, Vargas H, Posso H, Terry MB, Gissmann L, Schneider A, Mansukhani M, Rao PH, Murty VV. 2003b. Genetic analysis identifies putative tumor suppressor sites at 2q35-q36.1 and 2q36.3-q37.1 involved in cervical cancer progression. Oncogene 22:3489-3499.

Narayan G, Scotto L, Neelakantan V, Kottoor SH, Wong AH, Loke SL, Mansukhani M, Pothuri B, Wright JD, Kaufmann AM, Schneider A, Arias-Pulido H, Tao Q, Murty VV. 2009. Protocadherin PCDH10, involved in tumor progression, is a frequent and early target of promoter hypermethylation in cervical cancer. Genes Chromosomes Cancer 48:983-992.

Ojesina AI, Lichtenstein L, Freeman SS, Pedamallu CS, ImazRosshandler I, Pugh TJ, Cherniack AD, Ambrogio L, Cibulskis K, Bertelsen B, Romero-Cordoba S, Trevino V, VazquezSantillan K, Guadarrama AS, Wright AA, Rosenberg MW, Duke F, Kaplan B, Wang R, Nickerson E, Walline HM, Lawrence MS, Stewart C, Carter SL, McKenna A, Rodriguez-Sanchez IP, Espinosa-Castilla M, Woie K, Bjorge L, Wik E, Halle MK, Hoivik EA, Krakstad C, Gabino NB, Gomez-Macias GS, Valdez-Chapa LD, Garza-Rodriguez ML, Maytorena G, Vazquez J, Rodea C, Cravioto A, Cortes ML, Greulich H, Crum CP, Neuberg DS, Hidalgo-Miranda A, Escareno CR, Akslen LA, Carey TE, Vintermyr OK, Gabriel SB, BarreraSaldana HA, Melendez-Zajgla J, Getz G, Salvesen HB, Meyerson M. 2014. Landscape of genomic alterations in cervical carcinomas. Nature 506:371-375.

Rao PH, Arias-Pulido H, Lu XY, Harris CP, Vargas H, Zhang FF, Narayan G, Schneider A, Terry MB, Murty VV. 2004. Chromosomal amplifications, $3 \mathrm{q}$ gain and deletions of 2q33-q37 are the frequent genetic changes in cervical carcinoma. BMC Cancer 4: 5

Ruiz de Almodovar C, Ruiz-Ruiz C, Rodriguez A, Ortiz-Ferron G, Redondo JM, Lopez-Rivas A. 2004. Tumor necrosis factorrelated apoptosis-inducing ligand (TRAIL) decoy receptor TRAIL-R3 is up-regulated by p53 in breast tumor cells through a mechanism involving an intronic p53-binding site. J Biol Chem 279:4093-4101.

Schiffman M, Rodriguez AC. 2008. Heterogeneity in CIN3 diagnosis. Lancet Oncol 9:404-406.

Scotto L, Narayan G, Nandula SV, Arias-Pulido H, Subramaniyam S, Schneider A, Kaufmann AM, Wright JD, Pothuri B, Mansukhani M, Murty VV. 2008a. Identification of copy number gain and overexpressed genes on chromosome arm 20q by an integrative genomic approach in cervical cancer: potential role in progression. Genes Chromosomes Cancer 47:755-765.

Scotto L, Narayan G, Nandula SV, Subramaniyam S, Kaufmann AM, Wright JD, Pothuri B, Mansukhani M, Schneider A, AriasPulido H, Murty VV. 2008b. Integrative genomics analysis of chromosome $5 \mathrm{p}$ gain in cervical cancer reveals target overexpressed genes, including Drosha. Mol Cancer 7:58

Sheridan JP, Marsters SA, Pitti RM, Gurney A, Skubatch M, Baldwin D, Ramakrishnan L, Gray CL, Baker K, Wood WI, Goddard AD, Godowski P, Ashkenazi A. 1997. Control of TRAIL-induced apoptosis by a family of signaling and decoy receptors. Science 277:818-821.

Shivapurkar N, Toyooka S, Toyooka KO, Reddy J, Miyajima K, Suzuki M, Shigematsu H, Takahashi T, Parikh G, Pass HI, Chaudhary PM, Gazdar AF. 2004. Aberrant methylation of trail decoy receptor genes is frequent in multiple tumor types. Int J Cancer 109:786-792.

Shivapurkar N, Sherman ME, Stastny V, Echebiri C, Rader JS, Nayar R, Bonfiglio TA, Gazdar AF, Wang SS. 2007. Evaluation of candidate methylation markers to detect cervical neoplasia. Gynecol Oncol 107:549-553.

Syrjanen KJ. 1996. Spontaneous evolution of intraepithelial lesions according to the grade and type of the implicated human papillomavirus (HPV). Eur J Obstet Gynecol Reprod Biol 65:45-53.

Takimoto R, El-Deiry WS. 2000. Wild-type p53 transactivates the KILLER/DR5 gene through an intronic sequence-specific DNA-binding site. Oncogene 19:1735-1743.

The ASC-US-LSIL Triage Study (ALTS) Group. 2003. Results of a randomized trial on the management of cytology interpretations of atypical squamous cells of undetermined significance. Am J Obstet Gynecol 188:1383-1392.

van Noesel MM, van Bezouw S, Salomons GS, Voute PA, Pieters R, Baylin SB, Herman JG, Versteeg R. 2002. Tumor-specific down-regulation of the tumor necrosis factor-related apoptosisinducing ligand decoy receptors DcR1 and DcR2 is associated with dense promoter hypermethylation. Cancer Res 62:21572161.

Waggoner SE. 2003. Cervical cancer. Lancet 361:2217-2225.

Williams SV, Platt FM, Hurst CD, Aveyard JS, Taylor CF, Pole JC, Garcia MJ, Knowles MA. 2010. High-resolution analysis of genomic alteration on chromosome arm $8 \mathrm{p}$ in urothelial carcinoma. Genes Chromosomes Cancer 49:642-659.

Xue W, Krasnitz A, Lucito R, Sordella R, Vanaelst L, CordonCardo C, Singer S, Kuehnel F, Wigler M, Powers S, Zender L, Lowe SW. 2008. DLC1 is a chromosome 8 p tumor suppressor whose loss promotes hepatocellular carcinoma. Genes Dev 22: 1439-1444.

Yang Q, Kiernan CM, Tian Y, Salwen HR, Chlenski A, Brumback BA, London WB, Cohn SL. 2007. Methylation of CASP8, DCR2, and HIN-1 in neuroblastoma is associated with poor outcome. Clin Cancer Res 13:3191-3197.

zur Hausen H. 2002. Papillomaviruses and cancer: from basic studies to clinical application. Nat Rev Cancer 2:342-350. 